\title{
Radiation properties of sources inside photonic crystals
}

\author{
Irfan Bulu,* Humeyra Caglayan, and Ekmel Ozbay \\ Department of Physics, Bilkent University, Bilkent, 06533 Ankara, Turkey
}

(Received 29 October 2002; revised manuscript received 14 February 2003; published 15 May 2003)

\begin{abstract}
In this work, we have experimentally studied the emission of radiation from a monopole source embedded in a photonic crystal. We have demonstrated the enhancement of radiation at the band edges and at the cavity modes including coupled cavity modes. Moreover, we have shown that the emission of radiation from a source depends on the group velocities of the modes and on the electric field intensities of the modes at the source location.
\end{abstract}

DOI: 10.1103/PhysRevB.67.205103

PACS number(s): 42.70.Qs

\section{INTRODUCTION}

The possibility of altering the spontaneous emission from radiation sources by means of electromagnetic mode distributions different from that of free space mode distribution was first predicted in 1946 by Purcell. ${ }^{1}$ This effect has been investigated experimentally in cavities with simple geometries, such as plane mirrors and spheres., ${ }^{2,3}$ Yablonovitch $^{4}$ proposed a different class of periodic structures, which we now call photonic crystals (PC's), that can be used to control spontaneous emission. PC's are structures with periodic dielectric constant variations. Periodic dielectric constant variations lead to electromagnetic band gaps (where there are no propagating electromagnetic modes) along certain directions in the crystal. ${ }^{5-8}$ Absence of modes along certain directions can be used for various applications, such as microscopic lasers, ${ }^{9}$ resonant antennas, ${ }^{10}$ and optical switches. ${ }^{11}$

Most of the work devoted to the PC's up to now have focused on the transmission and reflection properties of PC's. However, this provides little insight into how a PC modifies the emission properties of a radiating source. The effect of PC's on the radiation properties of sources have been theoretically investigated by several authors. ${ }^{13-16,12}$ Interesting phenomena such as photon-atom bound dressed states, ${ }^{16}$ nonexponential decay of spontaneous emission near the band edge, ${ }^{15}$ and strong inhibition of emission and enormous enhancement of radiation ${ }^{12}$ have been reported. These works have revealed that PC's may provide far-reaching control over the spontaneous emission and the radiation dynamics of sources. For instance, spontaneous emission can be enhanced, suppressed or attenuated in all or certain directions by changing the density of modes. ${ }^{12}$

Surprisingly, only a few experiments have been reported on the radiation properties of localized sources inside PC's. Preparation of well defined systems is the major problem for such experiments. Some experiments have been performed to investigate the emission properties of dye molecules embedded inside PC's. ${ }^{17,18}$ These experiments have led to different interpretations and are still subject to discussion..$^{19,20}$ On the other hand, it has been suggested that similar effects such as nonexponential decay rates, ${ }^{21}$ inhibition of radiation, ${ }^{22}$ enormous enhancement at the band edges ${ }^{12}$ and cavity modes ${ }^{23}$ can be observed at longer wavelength scales.

The effect of PC's on the radiation of a localized source is encapsulated in local density of states (LDOS), which de- pends on position and frequency. For a PC, LDOS is proportional to the amplitude of the electric field at the position where the source is located. On the other hand, it is inversely proportional to the group velocity of the mode, $\partial \omega / \partial k$, at which the source radiates. ${ }^{24}$ In this sense, PC's provide enormous control over the radiation of a source, since for a PC it is possible to find positions with high or low electric-field amplitude and a mode with low or high group velocity. In this paper, we report the radiation properties of a finite-size line source, which we will call hereafter a monopole source, embedded inside a PC.

\section{ENHANCEMENT OF RADIATION AND REDUCED GROUP VELOCITIES}

In our experiments, we have studied the emission of microwave radiation from a monopole source located inside a PC and a monopole source located inside various defect structures created inside a PC. The monopole source is obtained by removing $0.5 \mathrm{~cm}$ of the cladding from a coaxial cable and leaving the metal part. The monopole source is then excited by the network analyzer. An HP-8510C vector network analyzer is used in our measurements. HP-8510C network analyzer is capable of measuring both intensity and phase. The phase information obtained from the measurement is used to determine the delay time or the photon lifetime. The photon lifetime is defined as $\tau_{p}=\partial \varphi / \partial \omega$. Here, $\varphi$ is the net phase difference between the phase of the electromagnetic (EM) waves propagating inside the photonic crystal and the phase of the EM waves propagating in free space for a total crystal length of $\mathrm{L}$. The photon lifetime corresponds to the propagation time of the EM waves inside the photonic crystal. ${ }^{25}$ Hence, group velocity is inversely proportional to the photon lifetime. The photon lifetime and its physical interpretations have been rigorously studied by Ohtaka et al. $^{26-29}$ It has been shown that the optical density of modes is directly proportional to the photon lifetime. ${ }^{29}$

\section{A. Enhanced emission from the monopole at the band edges}

For a finite PC, the group velocities of the modes at the band edges are expected to be small ${ }^{30-32}$ and correspondingly, the photon lifetimes are expected to be high. Hence it follows that LDOS is high at the band edge modes as LDOS is inversely proportional to the group velocity. 


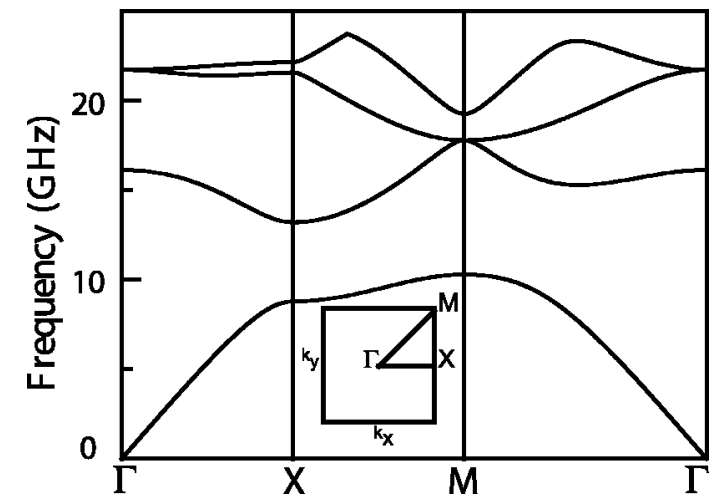

FIG. 1. Band structure of the corresponding infinite photonic crystal. The first four TM polarized bands have been calculated by plane wave expansion.

The $\mathrm{PC}$ used in the experiments is a $25 \times 25$ square array of cylindrical alumina rods whose radius is $1.55 \mathrm{~mm}$ and dielectric constant is 9.61. The separation between the center of rods along lattice vectors is $a=11 \mathrm{~mm}$. The structure that we have used in our experiments has a filling fraction of 0.062. The photonic band structure and the corresponding eigenvectors are calculated by plane-wave expansion method. Figure 1 shows the photonic band structure of the corresponding infinite photonic crystal for the first four TM polarized (electric field is parallel to the axis of the rods) bands. According to the band structure calculations the lower band edge of the first band gap along $\Gamma-X$ is at $8.70 \mathrm{GHz}$ and, the upper band edge of the first band gap along $\Gamma-X$ is at $13.20 \mathrm{GHz}$.

We have measured the transmission properties and the photon lifetime of the PC along the $\Gamma-X$ direction. The transmission measurement [Fig. 2(a)] indicates that the lower edge of the first band gap along the $\Gamma-X$ direction is around 8.7 $\mathrm{GHz}$ and the upper edge is around $13.2 \mathrm{GHz}$. These values are in agreement with the photonic band structure (Fig. 1). The measured photon lifetimes around the lower band edge and the upper band edge are shown in Figs. 2(b) and 2(c), respectively. As shown in both figures, the delay time significantly increases near the band edges. The photon lifetime near the lower edge is $12.9 \mathrm{nsec}$, which is $14 \times$ larger than the time required for the em waves to propagate along the structure $(0.92 \mathrm{nsec})$. So, the $\mathrm{PC}$ reduces the group velocity of light at this frequency by a factor of 14 . For the upper edge, the lifetime is $19.2 \mathrm{nsec}$, which corresponds to a 22 times reduction for the speed of light.

The enhancement factor for the EM waves emitted from a monopole source along the $\Gamma-X$ direction is measured for various source locations inside the center cell of the photonic crystal. The enhancement factor is defined as the ratio of the intensity of the EM waves emitted from a source located inside the photonic crystal to the intensity of the EM waves emitted from a source in free space. Figures 3(a) and (b) show the enhancement factors measured at various source locations inside the center unit cell for frequencies near the lower edge and the upper edge, respectively. The source locations are shown in Fig. 3(c). Although enhancement of radiation is expected for the band edge modes, the enhance-

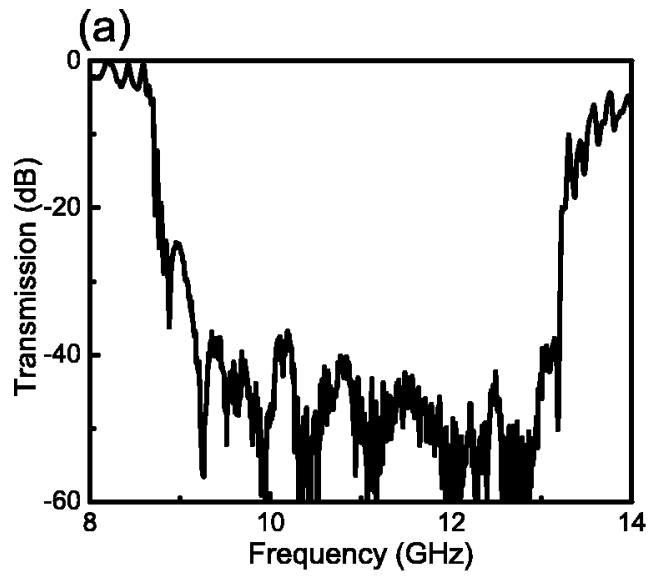

(b)

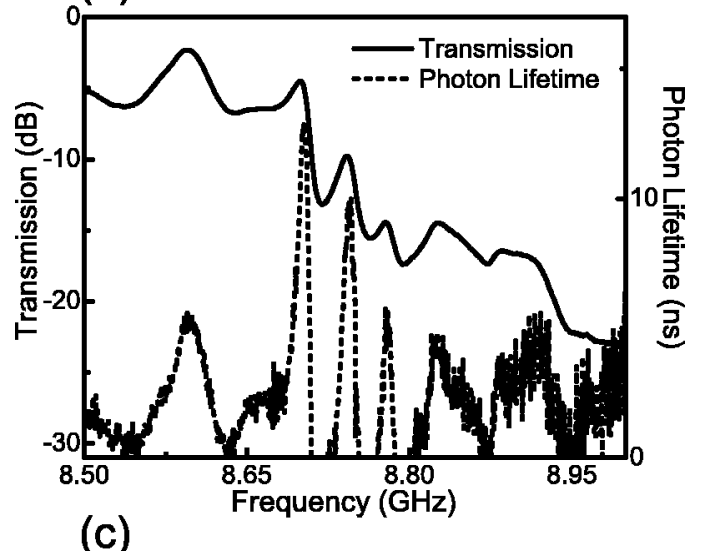

(c)

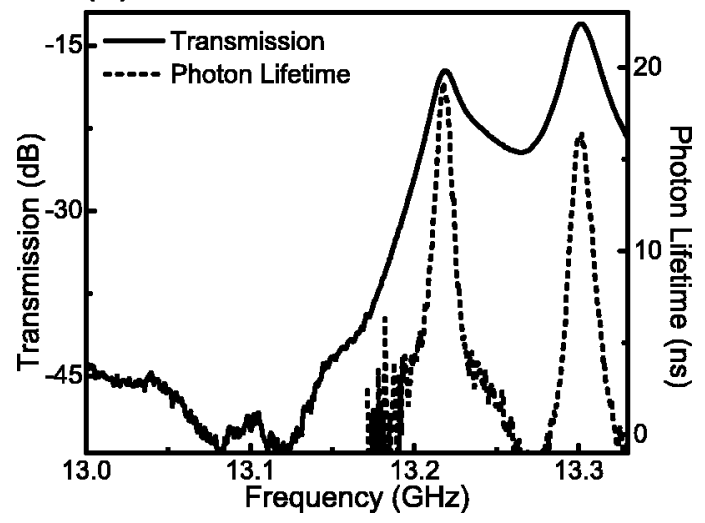

FIG. 2. (a) Transmission along $\Gamma-X$ between 8 and $14 \mathrm{GHz}$. (b) Solid curve represents transmission and dashed curve represents photon lifetime for the lower band edge along $\Gamma-X$ direction. (c) Solid curve represents transmission and dashed curve represents photon lifetime for the upper band edge along the $\Gamma-X$ direction.

ment factor is not the same at all of the source locations. The enhancement factors presented in Figs. 3(a) and (b) show that the enhancement factor decreases for the lower band edge and increases for the upper band edge as we move the source away from the center rod. This can be explained by the fact that the emission of radiation depends on the intensity of the modes at the source location. The modes of the lower band edge are dielectric modes, while the modes of the upper band edge are air modes. Hence, as we move the source away from the center rod, LDOS and correspond- 


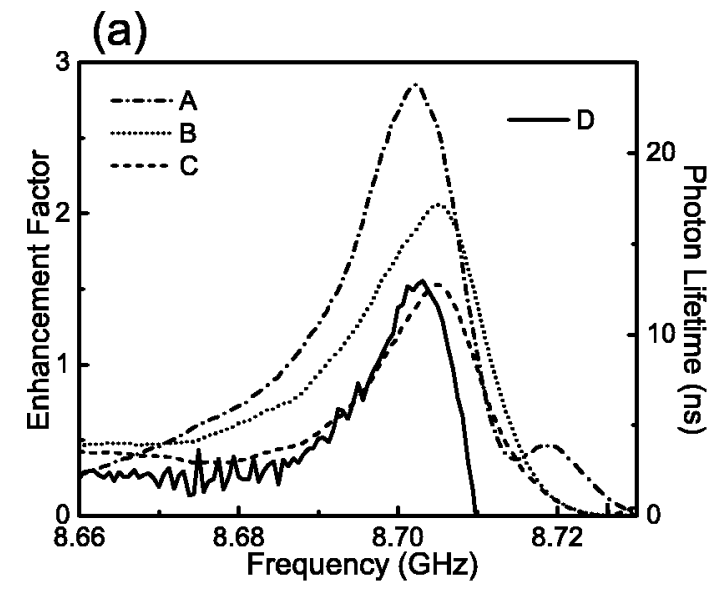

(b)

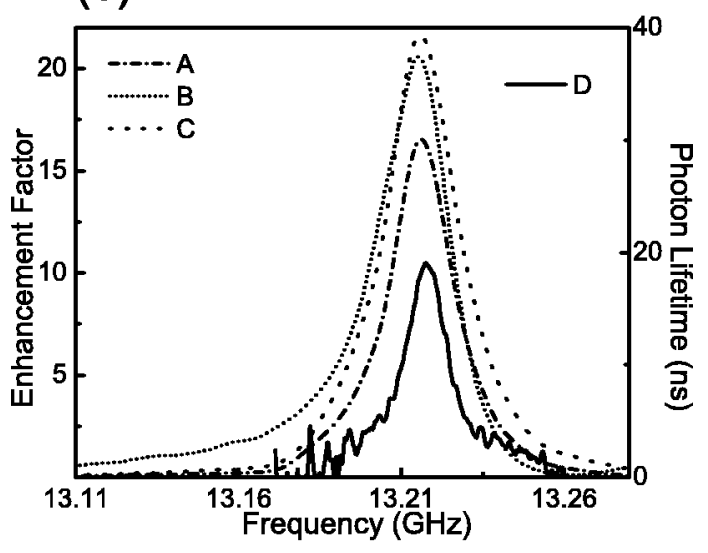

(c)

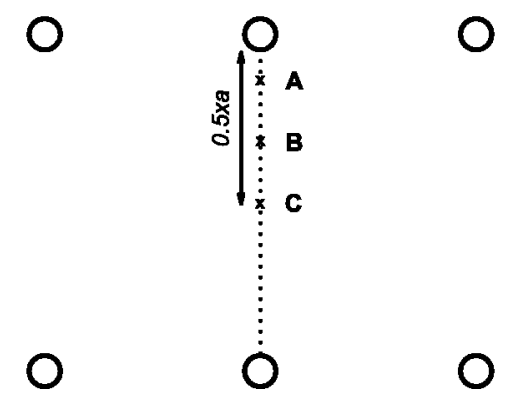

FIG. 3. (a) Enhancement factor near the lower band edge along $\Gamma-X$ for a source located at A: $0.1 \times a, \mathrm{~B}: 0.3 \times a, \mathrm{C}: 0.5 \times a$ away from the center rod. D represents the measured photon lifetime (b) Enhancement factor near the upper band edge along $\Gamma-X$ for a source located at A: $0.1 \times a, \mathrm{~B}: 0.3 \times a, \mathrm{C}: 0.5 \times a$ away from the center rod. D represents the measured photon lifetime. (c) A, B, and $\mathrm{C}$ show the source locations that are used in Figs. 3(a) and (b).

ingly, the enhancement factor is expected to decrease for the lower band edge and increase for the upper band edge. We also observe that the maximum enhancement factor for the upper band edge is higher than the enhancement factor for the lower band edge. This can be attributed to two major factors. First, for the lower band edge the electric field intensity of the mode is high inside the high dielectric material. Since the high dielectric region of the photonic crystal is not experimentally accessible, the possible highest enhancement factor cannot be measured for the lower band edge. Second,

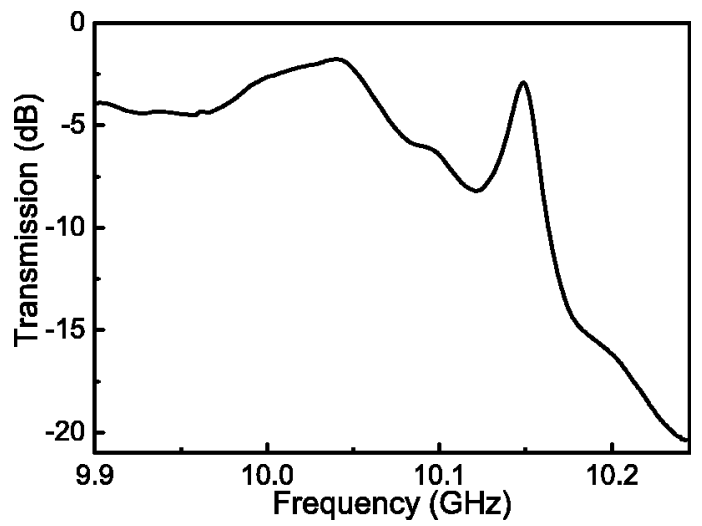

FIG. 4. Solid curve represents the transmission along the $\Gamma-M$ direction.

the higher bands are flatter than the lower bands. This results in lower group velocities for the upper band edge for a finite photonic crystal. ${ }^{33}$ Hence we expect LDOS and enhancement factor to be higher for the upper band edge when compared to the lower band edge.

The photonic band structure (Fig. 1) shows that at the lower band edge frequency along the $\Gamma-X$ direction there are modes along other propagation directions, such as the equal frequency mode along $\Gamma-M$ direction. So, a monopole source placed inside the photonic crystal can also radiate into these other modes. On the other hand, at the bandedge frequency along the $\Gamma-M$ direction, there are no such equal frequency modes along different propagation directions. Hence we expect to observe higher enhancement factors for the frequencies near the lower band edge along $\Gamma-M$ when compared to the $\Gamma-X$ direction. We experimentally check this argument by measuring the enhancement factor near the lower edge of the first band gap along the $\Gamma-M$ direction. The lower edge along the $\Gamma-M$ direction is around $10.15 \mathrm{GHz}$ (Fig. 4). Figure 5 shows the enhancement factor along $\Gamma-M$ for various source locations inside the center cell of the photonic crystal around $10.15 \mathrm{GHz}$. A maximum enhancement factor of 4.2 is observed at the lower band-edge frequency along the $\Gamma-M$ direction, which is higher than the maximum enhancement factor measured for the $\Gamma-X$ direction.

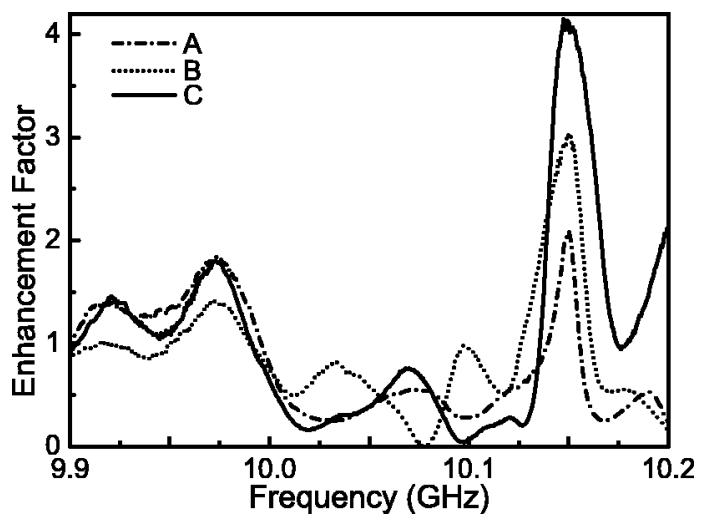

FIG. 5. Enhancement factor near the lower band edge along $\Gamma-M$ for a source located at A: $0.5 \times a, \mathrm{~B}: 0.3 \times a, \mathrm{C}: 0.1 \times a$ away from the center rod. 
(a)

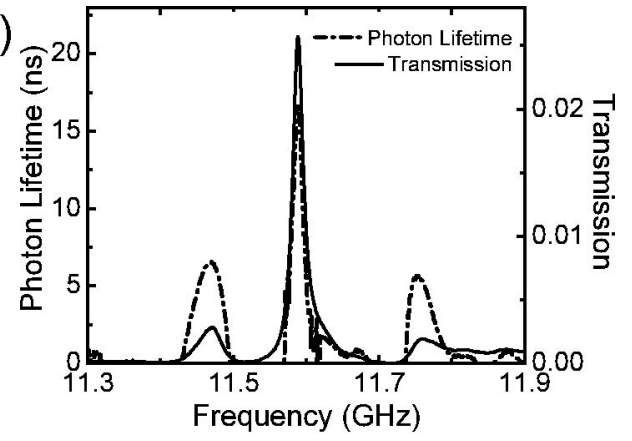

(b)

(c)

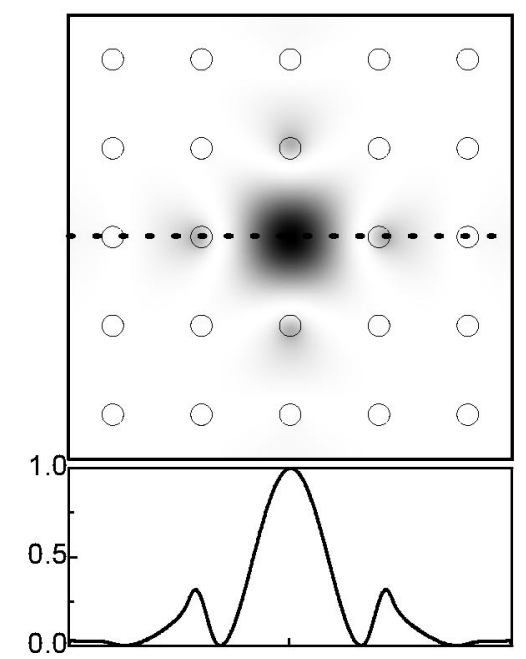

FIG. 6. (a) Solid curve represents transmission and dashed curve represents photon lifetime for the cavity mode. (b) Contour plot of electric-field intensity for the cavity mode. Electric-field intensity has been calculated by plane-wave expansion method. A $5 \times 5$ supercell has been used in the calculation. (c) Intensity of the electric field for the cavity mode along the cross section shown with dotted line in (a).

\section{B. Monopole inside a cavity}

In the previous sections, we have emphasized the dependence of LDOS and enhancement factors on the intensity of the modes at the source locations and on the group velocities of the modes. In this sense, single cavity structures are promising structures for the control of emission from radiation sources, as the intensity of the cavity mode is localized and the group velocity for the cavity mode is reduced. Single cavity structures have been investigated by several authors. ${ }^{34-38}$ A variety of applications utilizing cavity structures have been proposed, such as single-mode light-emitting diodes, ${ }^{39}$ resonant antennas, ${ }^{10}$ optical filters, ${ }^{40}$ and resonant cavity enhanced detectors. ${ }^{41}$

We have removed the center rod from a $9 \times 9$ square array of alumina rods to create the single cavity structure. The measured transmission and the delay data for the single cavity structure are shown in Fig. 6(a). The transmission data indicates that the defect mode is around $11.6 \mathrm{GHz}$ with a peak transmission of 0.025 . The photon lifetime measurement shows an increase in the photon lifetime by a factor of 48. This also means that at the defect mode the group velocity is 48 times smaller when compared to the electromagnetic waves propagating in free space. In addition to the reduced (a)

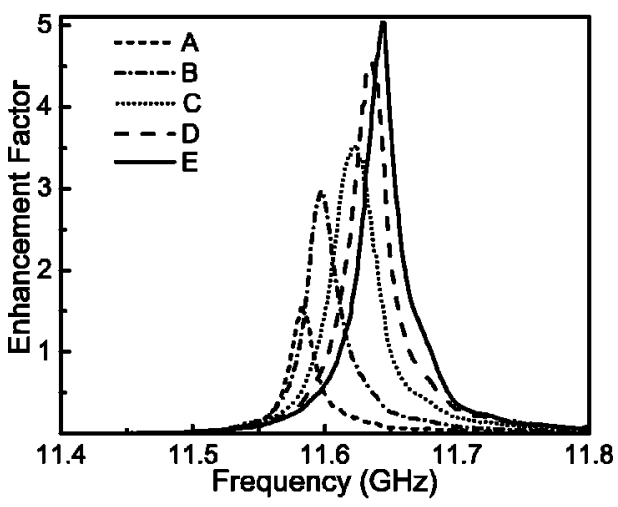

(b)

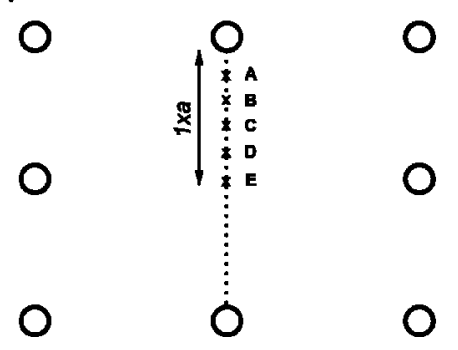

FIG. 7. (a) Enhancement factor for a source located inside a cavity A: $0.2 \times a, \mathrm{~B}: 0.4 \times a, \mathrm{C}: 0.6 \times a, \mathrm{D}: 0.8 \times a, \mathrm{E}: 1 \times a$ away from the rod. (b) A, B, C, D, and E show the source locations that are used in (a).

group velocity, the computed mode profile in Fig. 6(b) shows that the intensity of the cavity mode is localized inside the cavity. Hence at the cavity mode we expect to observe high enhancement factors for a monopole source located inside

(a)

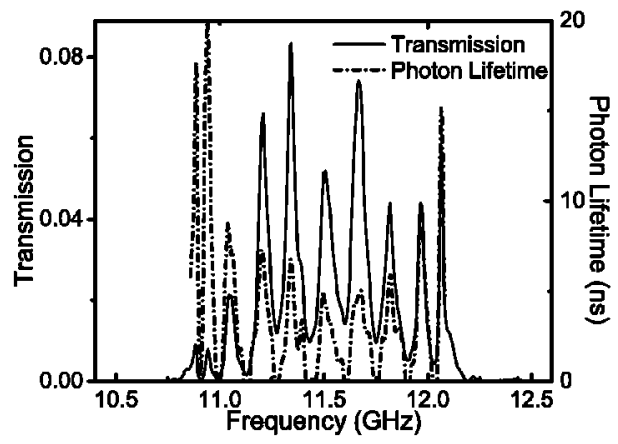

(b)

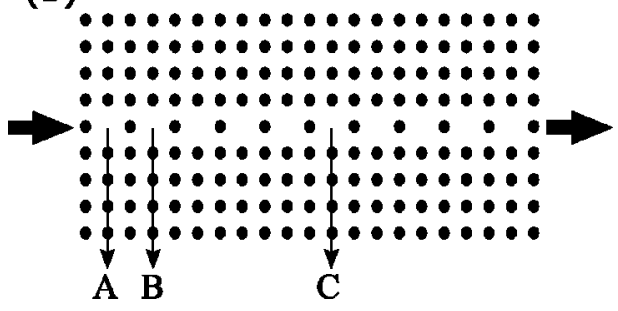

FIG. 8. (a) Solid curve represents transmission and dashed curve represents photon lifetime for the coupled cavity structure. (b) Schematics of coupled cavity structure. 


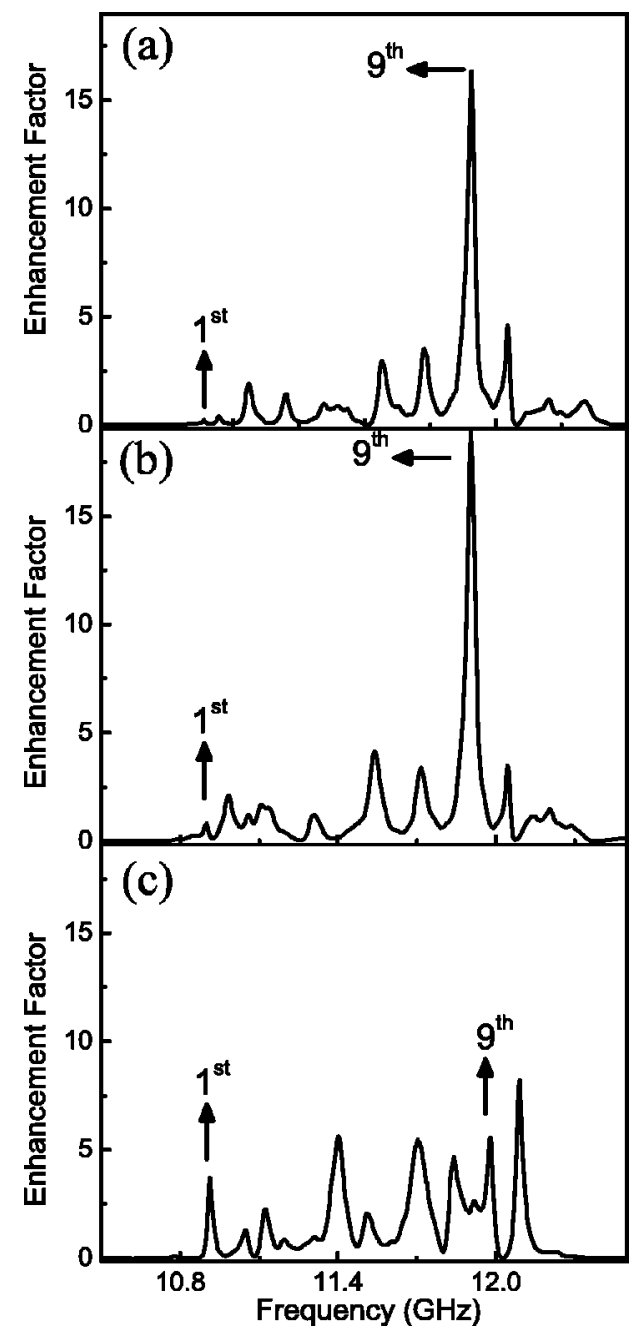

FIG. 9. Enhancement factor for a source located at the center of (a) cavity A, (b) cavity B, and (c) cavity C.

the cavity. Figure 7(a) shows the measured enhancement factor along the $\Gamma-X$ direction for various source locations inside the cavity. Enhancement factor measurements for the cavity structure show that as we move the source towards the center of the cavity, the cavity frequency shifts to higher frequencies. This is due to the modification of the cavity by the finite size of the radiation source. We also observe that as we move the source towards the center, the enhancement factor for the corresponding cavity mode increases. Maximum enhancement factor is obtained when the source is located at the center of the cavity. This can be explained by the LDOS picture. Since the intensity of the cavity mode increases towards the center of the cavity, LDOS is expected to increase as we move the radiation source towards the center of the cavity.

TABLE I. Measured enhancement factors at the first and ninth $\mathrm{CC}$ modes for a source placed at the center of cavities.

\begin{tabular}{lccc}
\hline \hline & Cavity A & Cavity B & Cavity C \\
\hline First mode & 0.24 & 0.80 & 3.72 \\
Ninth mode & 16.30 & 18.80 & 5.55 \\
\hline \hline
\end{tabular}

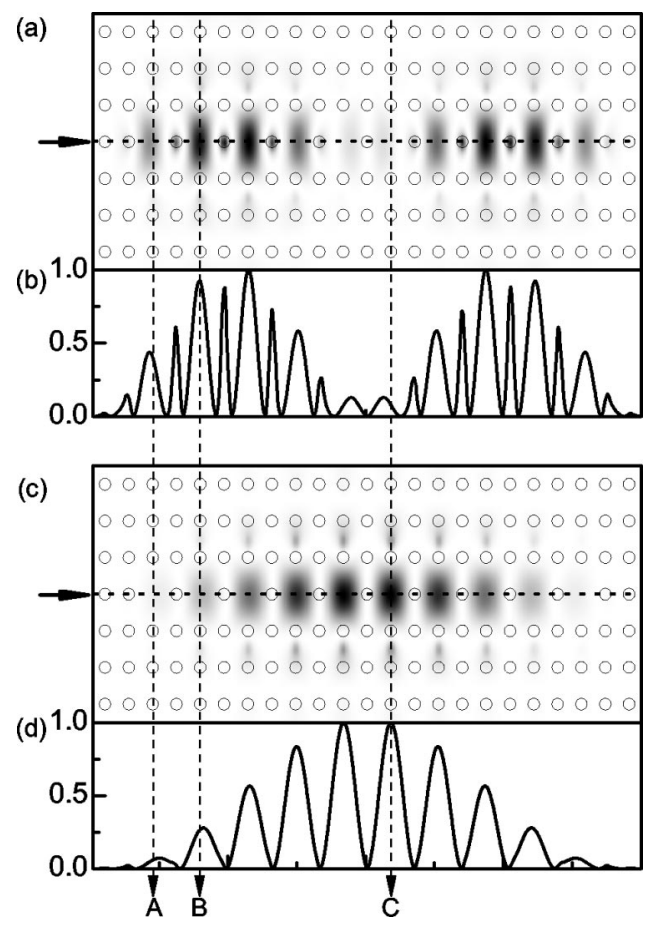

FIG. 10. (a) Contour plot of electric-field intensity for the ninth $\mathrm{CC}$ mode. Electric-field intensity was calculated by the plane-wave expansion method using a $23 \times 5$ supercell. (b) Electric-field intensity for the ninth $\mathrm{CC}$ mode along the cross section shown with dotted line in Fig. 10(a). The cross section is along the direction of propagation and crosses the perpendicular direction at the center of the cavities. (c) Contour plot of the electric-field intensity for the first CC mode. (d) Electric-field intensity for the first cavity along the cross section shown with a dotted line in Fig. 10(c). The cross section is along the direction of propagation and crosses the perpendicular direction at the center of the cavities.

\section{Monopole inside coupled cavity structure}

Coupled cavity (CC) structures have been investigated by many authors for their wave guiding properties. ${ }^{42-47}$ These structures provide a different mechanism for electromagnetic wave propagation. In CC structures, the mechanism for electromagnetic wave propagation is the hopping of photons due to the interaction between the neighboring evanescent cavity modes. ${ }^{25}$ For CC structures, reduced group velocities for the $\mathrm{CC}$ modes have been reported. ${ }^{25}$ Also the electric field intensity of CC modes are localized in the coupled cavities.

The CC structure that we have used for our experiments is created by introducing ten single cavities to the 11th column of a $21 \times 21$ square array of alumina rods. A single cavity is obtained by removing one rod from the perfect crystal. Each cavity is separated from the neighboring cavity by one rod. Figure 8(b) shows the details of the CC structure. The transmission and photon lifetime data presented in Fig. 8(a) shows that there are ten cavity modes, which are equal to the number of cavities. At the CC modes, transmission is high compared to the transmission through the photonic crystal without the coupled cavities. Transmissions as high as $10 \%$ have been observed at the $\mathrm{CC}$ modes. We also observe that the photon lifetime is increased at the CC modes. The increase in the photon lifetime indicates that the group veloci- 
ties of the $\mathrm{CC}$ modes are reduced when compared to the EM waves propagating in free space. For the first two CC modes (CC modes are numbered in order of increasing frequency), we observed relatively low transmission compared to other $\mathrm{CC}$ modes. This low transmission indicates that coupling to these modes is poor.

Due to the strong interaction between the cavities, CC structures drastically modify the spontaneous emission of radiation. We measured the enhancement factor when the monopole source is located at the center of the first cavity (cavity A), the second cavity (cavity B), and the sixth cavity (cavity C). The measured enhancement factors when the source is placed at the center of cavity A, cavity B, and cavity $\mathrm{C}$ are shown in Figs. 9(a)-(c), respectively. The enhancement factors for the first and ninth $\mathrm{CC}$ modes are given in Table I. We observe that when the source is placed either at cavity A or cavity B, ninth CC mode has the highest enhancement factors, while the first $\mathrm{CC}$ mode has the lowest enhancement factor compared to the other CC modes. At cavity A and cavity B, enhancement factors of 16.3 and 18.8 have been observed respectively (Table I). On the other hand, Fig. 9(c) and Table I show that when we place the source at cavity $\mathrm{C}$, the enhancement factor for the first $\mathrm{CC}$ mode is higher for cavity $\mathrm{C}$, when compared to cavities A and $\mathrm{B}$. This can be explained by the fact that the enhancement factor depends on the intensity of electric field at the source location. Since the intensity of the electric field for the ninth CC mode shown in Figs. 10(a) and (b) is high at the centers of cavity A and cavity B, we expect higher enhancement factors for this CC mode when the source is placed at cavity A or cavity B. On the other hand, intensity of the electric field for the first CC mode shown in Figs. 10(c) and (d) is high at the center of cavity C. Hence when we place the source at cavity $\mathrm{C}$, we expect the first mode to have a higher enhancement factor compared to the enhancement factors obtained for this mode when the source is placed at either cavity A or cavity B.

\section{CONCLUSION}

In summary, we studied the emission of radiation from a monopole source located inside a photonic crystal and inside various defect structures created in a photonic crystal. We showed the enhancement of radiation along with reduced group velocities at the band edges. Moreover, we experimentally demonstrated the enhancement of radiation at a single cavity mode and at the $\mathrm{CC}$ modes. At the single cavity and $\mathrm{CC}$ modes, low group velocities along with high electricfield intensities result in increased enhancement factors. The enhancement factor for a source depends on the group velocity of the mode and on the intensity of the electric field at the source location.

\section{ACKNOWLEDGMENTS}

This work was supported by a grant from European Union under EU-DALHM project and by a grant from NATO (Grant No. SfP971970). Also, the authors would like to thank Professor Ezel K. Shaw for her comments during the preparation of the manuscript.
*Electronic address: irfan@fen.bilkent.edu.tr

${ }^{1}$ E. M. Purcell, Phys. Rev. 69, 681 (1946).

${ }^{2}$ P. Goy, J. M. Raimond, M. Gross, and S. Haroche, Phys. Rev. Lett. 50, 1903 (1983).

${ }^{3}$ P. Dobiasch and H. Dehmelt, Ann. Phys. (Paris) 10, 825 (1985).

${ }^{4}$ E. Yablonovitch, Phys. Rev. Lett. 58, 2059 (1987).

${ }^{5}$ K. M. Ho, C. T. Chan, and C. M. Soukoulis, Phys. Rev. Lett. 65 , 3152 (1990).

${ }^{6}$ E. Ozbay, E. Michel, G. Tuttle, R. Biswas, M. Sigalas, and K.-M. Ho, Appl. Phys. Lett. 64, 2059 (1994).

${ }^{7}$ J. D. Joannopoulos, R. D. Meade, and J. N. Winn, Photonic Crystal: Molding the Flow of Light (Princeton University Press, Princeton, NJ, 1995).

${ }^{8}$ E. Ozbay, J. Opt. Soc. Am. B 13, 1945 (1996).

${ }^{9}$ O. Painter, R. K. Lee, A. Scherer, A. Yariv, J. D. O'Brien, P. D. Dapkus, and I. Kim, Science (Washington, DC, U.S.) 284, 1819 (1999).

${ }^{10}$ B. Temelkuran, M. Bayindir, E. Ozbay, R. Biswas, M. M. Sigalas, G. Tuttle, and K. M. Ho, J. Appl. Phys. 87, 603 (2000).

${ }^{11}$ K. Busch and S. John, Phys. Rev. Lett. 83, 967 (1999).

${ }^{12}$ R. Sprik, B. A. Van Tiggelen, and A. Legendijk, Europhys. Lett. 35, 265 (1996).

${ }^{13}$ A. A. Asatryan, K. Busch, R. C. McPhedran, L. C. Botten, C. Martijn de Stedrke, and N. A. Nicorovici, Phys. Rev. E 63, 046612 (2001).

${ }^{14}$ Virginie Lousse, Jean-Pol Vigneron, Xavier Bouju, and JeanMarie Vigoureux, Phys. Rev. B 64, 201104 (2001).
${ }^{15}$ Nipun Vats, Sajeev John, and Kurt Busch, Phys. Rev. A 65, 043808 (2002).

${ }^{16}$ S. John and T. Quang, Phys. Rev. A 50, 1764 (1994).

${ }^{17}$ M. Megens, J. E. G. J. Wijnhoven, A. Lagendijk, and W. L. Vos, J. Opt. Soc. Am. B 16, 1403 (1999).

${ }^{18}$ E. P. Petrov, V. N. Bogomolov, I. I. Kalosha, and S. V. Gaponenko, Phys. Rev. Lett. 81, 77 (1998).

${ }^{19}$ Mischa Megens, H. P. Schriemer, Ad Lagendijk, and Willem L. Vos, Phys. Rev. Lett. 83, 5401 (1999).

${ }^{20}$ E. P. Petrov, V. N. Bogomolov, I. I. Kalosha, and S. V. Gaponenko, Phys. Rev. Lett. 83, 5402 (1999).

${ }^{21}$ K. Busch, N. Vats, S. John, and B. C. Sanders, Phys. Rev. E 62, 4251 (2000).

${ }^{22}$ A. G. Galstyan, M. E. Raikh, and Z. V. Vardeny, Phys. Rev. B 62, 1780 (2000).

${ }^{23}$ J.-K. Hwang, H.-Y. Ryu, and Y.-H. Lee, Phys. Rev. B 60, 4688 (1999).

${ }^{24}$ K. Sakoda, Optical Properties of Photonic Crystals (SpringerVerlag, Germany, 2001).

${ }^{25}$ M. Bayindir and E. Ozbay, Phys. Rev. B 62, R2247 (2000).

${ }^{26}$ S. Yano, Y. Segawa, J. S. Bae, K. Mizuno, S. Yamaguchi, and K. Ohtaka, Phys. Rev. B 66, 075119 (2002).

${ }^{27}$ T. Kondo, M. Hangyo, S. Yamaguchi, S. Yano, Y. Segawa, and K. Ohtaka, Phys. Rev. B 66, 033111 (2002).

${ }^{28}$ K. Ohtaka, Y. Suda, S. Nagano, T. Ueta, A. Imada, T. Koda, J. S. Bae, K. Mizuno, S. Yano, and Y. Segawa, Phys. Rev. B 61, 5267 (2000). 
${ }^{29}$ K. Ohtaka, J. Lightwave Technol. 17, 2161 (1999).

${ }^{30}$ A. Imhof, W. L. Vos, R. Sprik, and A. Lagendijk, Phys. Rev. Lett. 83, 2942 (1999).

${ }^{31}$ K. Inoue, N. Kawai, Y. Sugimoto, N. Carlsson, N. Ikeda, and K. Asakawa, Phys. Rev. B 65, 121308 (2002).

${ }^{32}$ T. Aoki, M. Wada Takeda, J. W. Haus, Z. Yuan, M. Tani, K. Sakai, N. Kawai, and K. Inoue, Phys. Rev. B 64, 045106 (2001).

${ }^{33}$ D.-Y. Jeong, Y. H. Ye, and Q. M. Zhang, J. Appl. Phys. 92, 4194 (2002).

${ }^{34}$ E. Ozbay, G. Tuttle, M. Sigalas, C. M. Soukoulis, and K. M. Ho, Phys. Rev. B 51, 13961 (1995).

${ }^{35}$ M. M. Sigalas, K. M. Ho, R. Biswas, and C. M. Soukoulis, Phys. Rev. B 57, 3815 (1998).

${ }^{36}$ C. J. M. Smith, R. M. De La Rue, M. Rattier, S. Olivier, H. Benisty, C. Weisbuch, T. F. Krauss, R. Houdré, and U. Oesterle, Appl. Phys. Lett. 78, 1487 (2001).

${ }^{37}$ T. Ochiai and J. Sänchez-Dehesa, Phys. Rev. B 65, 245111 (2002).

${ }^{38}$ S. Lan, S. Nishikawa, Y. Sugimoto, N. Ikeda, K. Asakawa, and H. Ishikawa, Phys. Rev. B 65, 165208 (2002).

${ }^{39}$ I. Schnitzer, E. Yablonovitch, A. Scherer, and T. J. Gmitter, in
Photonic Band Gap and Localization, Vol. 308 of NATO Advanced Study Institute, Series B: Physics, edited by C. M. Soukoulis (Kluwer Academic Publishers, The Netherlands, 1993), pp. 369-378.

${ }^{40}$ J. D. Joannopoulos, in Photonic Band Gap Materials, Vol. 315 of NATO Advanced Study Institute, Series E: Applied Sciences, edited by C. M. Soukoulis (Kluwer Academic Publishers, The Netherlands, 1996), pp. 1-21.

${ }^{41}$ B. Temelkuran, E. Ozbay, J. P. Kavanaugh, G. Tuttle, and K. M. Ho, Appl. Phys. Lett. 72, 2376 (1998).

${ }^{42}$ N. Stefanou and A. Modinos, Phys. Rev. B 57, 12127 (1998).

${ }^{43}$ A. Yariv, Y. Xu, R. K. Lee, and A. Scherer, Opt. Lett. 24, 711 (1999).

${ }^{44}$ Mehmet Bayindir, E. Cubukcu, I. Bulu, and E. Ozbay, Phys. Rev. B 63, 161104 (2001).

${ }^{45}$ Sheng Lan, Satoshi Nishikawa, Hiroshi Ishikawa, and Osamu Wada, J. Appl. Phys. 90, 4321 (2001).

${ }^{46}$ M. Bayindir, B. Temelkuran, and E. Ozbay, Phys. Rev. B 61, R11855 (2000).

${ }^{47}$ V. Yannopapas, A. Modinos, and N. Stefanou, Phys. Rev. B 65, 235201 (2002). 